\title{
Two-loop corrections to Light-by-Light scattering in Supersymmetric QED*
}

\author{
T. Binoth ${ }^{a}$, E. W. N. Glover ${ }^{b}$, P. Marquard ${ }^{c}$ and J. J. van der Bij ${ }^{c}$ \\ ${ }^{a}$ Department of Physics and Astronomy, \\ The University of Edinburgh, EH9 3JZ Edinburgh, Scotland \\ ${ }^{b}$ Department of Physics, University of Durham, Durham DH1 3LE, England \\ ${ }^{c}$ Fakultät für Physik, Albert-Ludwigs-Universität Freiburg, D-79104 Freiburg, \\ Germany \\ E-mail: binoth@ph.ed.ac.uk, E.W.N.Glover@durham.ac.uk, \\ marquard@physik.uni-freiburg.de, jochum@phyv1.physik.uni-freiburg.de
}

\begin{abstract}
We use an $n$-dimensional projection method to calculate helicity amplitudes for photon-photon scattering up to the two-loop level, for massless particles inside the loop. We confirm the recent standard model results found by Bern et al using another calculational method. We further apply the method to $N=1$ and $N=2$ supersymmetric QED. As predicted by the supersymmetric Ward identity, two of the independent amplitudes vanish. The remaining amplitude simplifies as the number of supersymmetries increases. For $N=2$ SUSY QED, only contributions of weight 4 remain.
\end{abstract}

KEYWORDS: two-loop; QED; SUSY; quantum electrodynamics; photon-photon.

\footnotetext{
${ }^{*}$ Work supported in part by the UK Particle Physics and Astronomy Research Council, by the EU Fifth Framework Programme 'Improving Human Potential', Research Training Network 'Particle Physics Phenomenology at High Energy Colliders', contract HPRN-CT-2000-00149 and by the DFG-Forschergruppe Quantenfeldtheorie, Computeralgebra und Monte-Carlo Simulation. We thank the British Council and German Academic Exchange Service for support under ARC project 1050. PM acknowledges the support of the German Academic Exchange Service.
} 


\section{Introduction}

Light-by-light scattering is a paradigm process in quantum field theory even if it is not yet of experimental relevance. ${ }^{1}$ The calculation of the process at the one-loop level, the first non vanishing order, was accomplished quite some time ago $[2,3,4,5,6]$. Gauge invariance and IR/UV finiteness leads to enormous cancellation mechanisms when individual Feynman graphs are summed up. As such the study of light-by-light scattering is an ideal testing ground for new methods relevant for loop calculations.

The computation of the higher order corrections to this process has been plagued by the difficulty of evaluating two-loop four point functions with four on-shell legs. At present it is not possible to include the mass of the particles circulating inside the loops. However, the complete set of integrals for planar on-shell two-loop graphs with massless internal particles is now known [7, 8, 9, 10, 11, 12, 13] together with a set of algorithms for reducing the tensor integrals down to the basis set of master integrals $[14,15,16]$. This technology has recently been applied to calculate the two-loop matrix elements for a wide range of $2 \rightarrow 2$ scattering processes [17, 18, 19, $20,21,22,23,1,24,25]$.

Recently the two-loop corrections for light-by-light scattering were calculated [1] by applying the helicity formalism at the two-loop level. The helicity formalism, which is strictly defined only in 4-dimensions greatly simplifies the calculation on the one-loop level. The generalisation to the two-loop case was done only recently [17, $23,1,24]$. In other cases $[18,19,20,21,22,25]$ the interference between two-loop and tree level amplitudes directly relevant for next-to-next-to-leading order calculations has been computed by working with $n$-dimensional kinematics only. This approach is not applicable in the case of four-photon scattering as a tree level operator simply does not exist.

In the present paper we follow another approach to calculate helicity amplitudes which in a certain sense interpolates between both methods. Namely by analysing the tensorial structure of the amplitude [2], valid to all orders in perturbation theory, we define projectors to isolate the coefficients of particular tensor structures. These projectors are defined in $n$-dimensions and therefore allow for a completely $n$-dimensional treatment of the computation of the tensor coefficients. The symmetry of the process dictates that there are only three independent coefficients. By fixing the helicity of the external photons, it is then straightforward to apply helicity methods to extract specific helicity amplitudes from the general tensor in terms of the three $n$-dimensional coefficients. The relation between the helicity amplitude and the tensor coefficients is independent of the order that the coefficients are computed at. It is also independent of whether or not the particles circulating in the loops are massive. As an explicit example, we focus on the circularly polarised amplitudes. Similar techniques can be applied to obtain linear polarised amplitudes. Note that

\footnotetext{
${ }^{1}$ See the nice review of the current experimental situation in Ref. [1].
} 
in defining the helicity amplitudes the external states are four-dimensional, however, the tensor coefficients are fully $n$-dimensional.

We apply the method to a fairly general class of Lagrangians containing photons, charged and neutral scalars and charged and neutral fermions with prescribed couplings. There are several gauge invariant classes and we list these contributions separately. We give explicit results for the $N=1$ and $N=2$ SUSY QED Lagrangians $[26,27,28]$. Although these unbroken supersymmetric theories have no direct phenomenological relevance, they have a theoretical interest of their own and have led to new insights in the possibilities of quantum field theory, in particular regarding its divergences. The presence of a supersymmetry leads to important cancellations between the bosonic and fermionic degrees of freedom. One of the best known consequences is the disappearance of quadratic divergences in $N=1$ supersymmetric theories. These divergences generally appear in renormalization constants and have no direct physical consequences. More interesting are cancellations or simplifications for physical quantities. These can be analysed on the basis of supersymmetric Ward identities $[29,30,31]$. In the case at hand these lead to the prediction that two of the independent helicity amplitudes vanish, a fact that we will verify in our calculation. The general analysis of supersymmetric Ward identities is a rather involved affair, due to the fact that there is no easily implementable supersymmetric regularization. The identification of a supersymmetry preserving regulator is beyond the scope of this paper. We therefore work in conventional dimensional regularisation which explicitly breaks supersymmetry by keeping $n-2$ polarizations for internal photons while the photino has only 2 degrees of freedom. However, because the quantities we calculate are infrared and ultraviolet finite, they are supersymmetric in the fourdimensional limit. There may be violations of supersymmetry of $\mathcal{O}(n-4)$, but they are not relevant in the four-dimensional limit.

While the simplifications in $N=1$ supersymmetry are already very interesting, stronger results are possible in the case of $N=2$ supersymmetry. For instance it is known that the beta function in $N=2$ super Yang-Mills theories is fully determined at the one-loop level. Since the infinite contributions that determine the beta function satisfy such strong non-renormalization theorems, one can also expect large cancellations for finite quantities. In order to check for such cancellations in the photon-photon scattering amplitude it is necessary to work at the two-loop level, since at the one-loop level there is no difference between $N=1$ and $N=2$ supersymmetry. We will indeed find an interesting pattern of simplifications taking place going up in the number of supersymmetries. Similar simplifications for certain supersymmetric higher loop amplitudes were already reported in the case of $N=4$ Super-Yang-Mills [32] and $N=8$ Supergravity [33].

Our paper is organised as follows. In Sec. 2 we recall the Lagrangian for Supersymmetric QED and review the particle content of the theory. The relevant Feynman rules are collected in Appendix A. The general tensor structure for light-by-light 
scattering is detailed in Sec. 3, together with the constraints from transversality of the external polarisation vectors, Bose symmetry and the gauge Ward identities that reduce the number of independent tensor coefficients to three. The general tensor is mapped onto circularly polarised helicity amplitudes in Sec. 3.1. We explicitly show how each of the three independent helicity amplitudes can be written in terms of the three $n$-dimensional tensor coefficients. In Sec. 3.2 we introduce projection operators that can be applied to the full tensor to isolate any of the tensor coefficients. A discussion of the Supersymmetric Ward identities is given in Sec. 4.1 while the results of an explicit calculation of the one- and two-loop helicity amplitudes are given in Secs. 4.2 and 4.3 respectively. The individual contributions to the two-loop amplitudes from the gauge invariant sub-classes of diagrams are listed in Appendix B. Finally our findings are summarised in Sec. 5.

\section{The SUSY QED Lagrangian}

$N=1$ Supersymmetric QED [26, 27] is an abelian gauge theory containing a vector multiplet $\left(A^{\mu}, \lambda_{\alpha}, \bar{\lambda}^{\dot{\alpha}}\right)$ containing the vector photon field and the Majorana photino and two chiral multiplets $\left(\psi_{L}^{\alpha}, \phi_{L}^{-}\right)$and $\left(\psi_{R}^{\alpha}, \phi_{R}^{+}\right)$with charge $Q_{L}=-1$ and $Q_{R}=+1$, each consisting of a Weyl spinor and a scalar field representing the electron and scalar electron matter fields. The $N=1$ SUSY QED Lagrangian with massless matter fields is given by,

$$
\begin{aligned}
\mathcal{L}_{\mathrm{SQED}}^{N=1}= & -\frac{1}{4} F_{\mu \nu} F^{\mu \nu}+\frac{1}{2} \tilde{\gamma}_{1} i \gamma^{\mu} \partial_{\mu} \tilde{\gamma}_{1} \\
& +\left|D_{\mu} \phi_{L}^{-}\right|^{2}+\left|D_{\mu}^{\dagger} \phi_{R}^{+}\right|^{2}+\bar{\Psi} i \gamma^{\mu} D_{\mu} \Psi \\
& +\sqrt{2} e\left(\bar{\Psi} P_{R} \tilde{\gamma}_{1} \phi_{L}^{-}-\phi_{R}^{+} \overline{\tilde{\gamma}_{1}} P_{R} \Psi+\text { h.c. }\right) \\
& -\frac{1}{2} e^{2}\left(\left|\phi_{L}^{-}\right|^{2}-\left|\phi_{R}^{+}\right|^{2}\right)^{2},
\end{aligned}
$$

where

$$
\Psi=\left(\begin{array}{c}
\psi_{L \alpha} \\
\bar{\psi}_{R}^{\dot{\alpha}}
\end{array}\right), \quad \tilde{\gamma}_{1}=\left(\begin{array}{c}
-i \lambda_{\alpha} \\
i \bar{\lambda} \dot{\alpha}
\end{array}\right),
$$

and where $D_{\mu}$ is the gauge covariant derivative and $F_{\mu \nu}$ is the field strength of the photon. This Lagrangian corresponds to the Wess-Zumino gauge where all unphysical fields are removed by gauge transformations.

$N=2$ Supersymmetric QED [26] contains in addition a chiral multiplet, a Majorana gaugino and a complex scalar photon, $\left(\psi_{\alpha}^{0}, \bar{\psi}^{0 \dot{\alpha}}, \phi^{0}\right)$. The $N=2$ SUSY QED Lagrangian with massless matter fields is given by,

$$
\begin{aligned}
\mathcal{L}_{\mathrm{SQED}}^{N=2}= & \mathcal{L}_{\mathrm{SQED}}^{N=1}+\frac{1}{2} \tilde{\gamma}_{2} i \gamma^{\mu} \partial_{\mu} \tilde{\gamma}_{2}+\left|\partial_{\mu} \phi^{0}\right|^{2} \\
& +\sqrt{2} e\left(\bar{\Psi} P_{L} \tilde{\gamma}_{2} \phi_{L}^{-}+\phi_{R}^{+} \overline{\tilde{\gamma}_{2}} P_{L} \Psi+\text { h.c. }\right)
\end{aligned}
$$




$$
\begin{aligned}
& +\sqrt{2} e\left(\bar{\Psi} P_{L} \Psi \phi^{0}+\phi^{0 *} \bar{\Psi} P_{R} \Psi\right) \\
& -2 e^{2}\left(\left|\phi_{L}^{-}\right|^{2}+\left|\phi_{R}^{+}\right|^{2}\right)^{2}\left|\phi^{0}\right|^{2} \\
& -\frac{1}{2} e^{2}\left(\left|\phi_{L}^{-}\right|^{2}+\left|\phi_{R}^{+}\right|^{2}\right)^{2}+\frac{1}{2} e^{2}\left(\left|\phi_{L}^{-}\right|^{2}-\left|\phi_{R}^{+}\right|^{2}\right)^{2}
\end{aligned}
$$

where

$$
\tilde{\gamma}_{2}=\left(\begin{array}{c}
\psi_{0 \alpha} \\
\bar{\psi}_{0}^{\dot{\alpha}}
\end{array}\right)
$$

Note that the $N=2$ supersymmetry leads to a $S U(2)$ symmetry under which the photinos and scalar electrons transform nontrivially. By writing them as doublets

$$
\left(\begin{array}{c}
\phi_{L}^{+} \\
\phi_{R}^{+}
\end{array}\right),\left(\begin{array}{c}
\tilde{\gamma}_{1} \\
\tilde{\gamma}_{2}
\end{array}\right)
$$

the photino-electron-selectron interactions can then be written as

$$
\mathcal{L}_{\gamma e \tilde{e}}=\sqrt{2} e\left(\phi_{L}^{-}, \phi_{R}^{-}\right)\left[\bar{\Psi} P_{R}\left(\begin{array}{ll}
1 & 0 \\
0 & 1
\end{array}\right)+\bar{\Psi} P_{L}\left(\begin{array}{cc}
0 & 1 \\
-1 & 0
\end{array}\right)\right]\left(\begin{array}{c}
\tilde{\gamma}_{1} \\
\tilde{\gamma}_{2}
\end{array}\right)+\text { h.c. }
$$

which is equivalent to the manifestly $\mathrm{SU}(2)$ invariant form presented in [28].

For completeness, the Feynman rules for these Lagrangians are given in Appendix A.

\section{The tensor structure of the four photon amplitude}

We consider the process where all particles are incoming

$$
\gamma\left(p_{1}, \lambda_{1}\right)+\gamma\left(p_{2}, \lambda_{2}\right)+\gamma\left(p_{3}, \lambda_{3}\right)+\gamma\left(p_{4}, \lambda_{4}\right) \rightarrow 0
$$

and photon $i$ carries momentum $p_{i}$ and has helicity $\lambda_{i}$. The amplitude has the form

$$
\mathcal{M}=\varepsilon_{1, \mu_{1}} \varepsilon_{2, \mu_{2}} \varepsilon_{3, \mu_{3}} \varepsilon_{4, \mu_{4}} \mathcal{M}^{\mu_{1} \mu_{2} \mu_{3} \mu_{4}}\left(p_{1}, p_{2}, p_{3}, p_{4}\right)
$$

where the scattering tensor $\mathcal{M}^{\mu_{1} \mu_{2} \mu_{3} \mu_{4}}$ has the following general decomposition

$$
\begin{aligned}
\mathcal{M}^{\mu_{1} \mu_{2} \mu_{3} \mu_{4}}= & A_{1} g^{\mu_{1} \mu_{2}} g^{\mu_{3} \mu_{4}}+A_{2} g^{\mu_{1} \mu_{3}} g^{\mu_{2} \mu_{4}}+A_{3} g^{\mu_{1} \mu_{4}} g^{\mu_{2} \mu_{3}} \\
& +\sum_{j_{1}, j_{2}=1}^{3}\left(B_{j_{1} j_{2}}^{1} g^{\mu_{1} \mu_{2}} p_{j_{1}}^{\mu_{3}} p_{j_{2}}^{\mu_{4}}+B_{j_{1} j_{2}}^{2} g^{\mu_{1} \mu_{3}} p_{j_{1}}^{\mu_{2}} p_{j_{2}}^{\mu_{4}}+B_{j_{1} j_{2}}^{3} g^{\mu_{1} \mu_{4}} p_{j_{1}}^{\mu_{2}} p_{j_{2}}^{\mu_{3}}\right. \\
& \left.\quad+B_{j_{1} j_{2}}^{4} g^{\mu_{2} \mu_{3}} p_{j_{1}}^{\mu_{1}} p_{j_{2}}^{\mu_{4}}+B_{j_{1} j_{2}}^{5} g^{\mu_{2} \mu_{4}} p_{j_{1}}^{\mu_{1}} p_{j_{2}}^{\mu_{3}}+B_{j_{1} j_{2}}^{6} g^{\mu_{3} \mu_{4}} p_{j_{1}}^{\mu_{1}} p_{j_{2}}^{\mu_{2}}\right) \\
& +\sum_{j_{1}, j_{2}, j_{3}, j_{4}=1}^{3} C_{j_{1} j_{2} j_{3} j_{4}} p_{j_{1}}^{\mu_{1}} p_{j_{2}}^{\mu_{2}} p_{j_{3}}^{\mu_{3}} p_{j_{4}}^{\mu_{4}} .
\end{aligned}
$$


Terms such as $\epsilon^{\mu_{1} \mu_{2} \mu_{3} \mu_{4}}$ are forbidden on the grounds of parity invariance. The functions $A_{i}, B_{j k}^{i}$ and $C_{i j k l}$ are functions of the Mandelstam variables, $s=\left(p_{1}+p_{2}\right)^{2}$, $t=\left(p_{2}+p_{3}\right)^{2}, u=\left(p_{1}+p_{3}\right)^{2}$ and the spacetime dimension $n$. They also depend on the mass of the electron and any other particles that may be involved in the scattering process. This decomposition is valid for arbitrary loop order since it is based solely on the Lorentz structure of the external particles. Altogether, there are 138 coefficients. However, many coefficients are irrelevant since they drop out when contracted with the photon polarisation vectors due to the transversality condition,

$$
\varepsilon_{j} \cdot p_{j}=0, \quad \text { for } j \in\{1,2,3,4\}
$$

This reduces the number of coefficients to 57 .

Bose symmetry of the external photons means that these coefficients are not independent. Requiring invariance under the exchange of any index pair $\left(j, \mu_{j}\right)$ where the first labels the external vector and the second is the Lorentz index of the corresponding polarisation vector reduces the number of independent functions to 11 .

The number of independent functions is further reduced by gauge symmetry. The related Ward identities read in an obvious notation,

$$
\mathcal{M}^{p_{1} \varepsilon_{2} \varepsilon_{3} \varepsilon_{4}}=\mathcal{M}^{\varepsilon_{1} p_{2} \varepsilon_{3} \varepsilon_{4}}=\mathcal{M}^{\varepsilon_{1} \varepsilon_{2} p_{3} \varepsilon_{4}}=\mathcal{M}^{\varepsilon_{1} \varepsilon_{2} \varepsilon_{3} p_{4}}=0
$$

Applying the gauge symmetry reduces the number of independent functions to three which we take to be,

$$
A_{1}(s, t, u), \quad B_{11}^{1}(s, t, u), \quad C_{2111}(s, t, u) .
$$

Once these functions are known, the full tensor can be reconstructed.

\subsection{Helicity amplitudes}

It is often convenient to express the amplitude in terms of the helicity structure of the scattering process. This can straightforwardly be achieved using the Lorentz structure of the tensor. In principle there are 16 helicity amplitudes - two polarisations for each photon. However, parity, time-reversal and Bose symmetry reduce this number to four which is further reduced to three by crossing symmetry. Note that the number of independent helicity amplitudes matches the number of independent functions describing the tensor. We take

$$
\mathcal{M}_{++++}, \quad \mathcal{M}_{+++-}, \quad \mathcal{M}_{++--}
$$

to be a basis from which the other helicity amplitudes can be reconstructed.

By specifying polarisation vectors for the external photons and applying them to the full tensor, we can derive the helicity amplitudes in terms of the three independent 
functions $A_{1}, B_{11}^{1}$ and $C_{2111}$. We find that, up to overall phases,

$$
\begin{aligned}
\mathcal{M}_{++++}= & A_{1}(s, t, u)+A_{1}(t, u, s)+A_{1}(u, t, s) \\
& -\frac{u^{2}}{4 t} B_{11}^{1}(s, t, u)-\frac{t(2 s+t)}{4 u} B_{11}^{1}(s, u, t)-\frac{u^{2}}{4 s} B_{11}^{1}(t, s, u) \\
& -\frac{s(t-u)}{4 u} B_{11}^{1}(t, u, s)+\frac{(2 s-t) t}{4 s} B_{11}^{1}(u, s, t)-\frac{s(s-2 t)}{4 t} B_{11}^{1}(u, t, s) \\
& +\frac{s(s-2 t) u}{8 t} C_{2111}(s, t, u)+\frac{s t(t-u)}{8 u} C_{2111}(s, u, t)+\frac{t^{2} u}{8 s} C_{2111}(t, s, u) \\
& -\frac{s t^{2}}{8 u} C_{2111}(t, u, s)+\frac{t u^{2}}{8 s} C_{2111}(u, s, t)+\frac{s u^{2}}{8 t} C_{2111}(u, t, s) \\
\mathcal{M}_{+++-}= & -\frac{u^{2}}{4 t} B_{11}^{1}(s, t, u)-\frac{t^{2}}{4 u} B_{11}^{1}(s, u, t)+\frac{u^{2}}{4 s} B_{11}^{1}(t, s, u) \\
& +\frac{s(t-u)}{4 u} B_{11}^{1}(t, u, s)+\frac{t^{2}}{4 s} B_{11}^{1}(u, s, t)+\frac{s(-t+u)}{4 t} B_{11}^{1}(u, t, s) \\
& +\frac{s(t-u) u}{8 t} C_{2111}(s, t, u)-\frac{s t(t-u)}{8 u} C_{2111}(s, u, t)-\frac{t^{2} u}{8 s} C_{2111}(t, s, u) \\
& +\frac{s t^{2}}{8 u} C_{2111}(t, u, s)-\frac{t u^{2}}{8 s} C_{2111}(u, s, t)+\frac{s u^{2}}{8 t} C_{2111}(u, t, s),
\end{aligned}
$$

and

$$
\begin{aligned}
\mathcal{M}_{++--}= & A_{1}(s, t, u)+A_{1}(t, u, s)+A_{1}(u, t, s) \\
& -\frac{u^{2}}{4 t} B_{11}^{1}(s, t, u)-\frac{t(2 s+t)}{4 u} B_{11}^{1}(s, u, t)-\frac{u^{2}}{4 s} B_{11}^{1}(t, s, u) \\
& -\frac{s(t-u)}{4 u} B_{11}^{1}(t, u, s)+\frac{(2 s-t) t}{4 s} B_{11}^{1}(u, s, t)-\frac{s(s-2 t)}{4 t} B_{11}^{1}(u, t, s) \\
& +\frac{s(s-2 t) u}{8 t} C_{2111}(s, t, u)+\frac{s t(t-u)}{8 u} C_{2111}(s, u, t)+\frac{t^{2} u}{8 s} C_{2111}(t, s, u) \\
& -\frac{s t^{2}}{8 u} C_{2111}(t, u, s)+\frac{t u^{2}}{8 s} C_{2111}(u, s, t)+\frac{s u^{2}}{8 t} C_{2111}(u, t, s) .
\end{aligned}
$$

Amplitudes for linearly polarised light can also be straightforwardly obtained from the general tensor and are given in terms of $A_{1}, B_{11}^{1}$ and $C_{2111}$.

Note that in deriving the helicity amplitudes, we have made no assumptions about how the functions $A_{1}, B_{11}^{1}$ and $C_{2111}$ are calculated. In the conventional approach to computing loop helicity amplitudes one has to define a description to deal with scalar products between loop momenta and polarisation vectors. Furthermore, we have made no assumptions about the masses of particles in the loops.

\subsection{Projection operators}

To calculate the independent functions $A_{1}, B_{11}^{1}$ and $C_{2111}$ it is convenient to define projection operators that (a) isolate the function and (b) saturate the free Lorentz 
indices. This latter point is important for practical calculations since it allows for the cancellation of reducible scalar products between loop momenta and external vectors in the Feynman diagram integrals. These reducible scalar products can be expressed in terms of inverse propagators and many cancellations already happen at this level. For example at the one-loop level, no tensor box integral needs to be evaluated since any loop momentum appearing in the numerator will immediately cancel one of the propagators. This reduces the complexity of the one-loop calculation enormously. For higher-loop or multi-leg applications such simplifications are highly appreciated.

To invert Eq. (3.3) and isolate the independent functions, it is useful to define the following tensors,

$$
\begin{aligned}
\mathcal{P}^{\mu \nu} & =g^{\mu \nu}-2 \sum_{j=1}^{3} \sum_{k=1}^{3} p_{j}^{\mu} H_{j k} p_{k}^{\nu} \\
\mathcal{R}_{j}^{\nu} & =2 \sum_{k=1}^{3} H_{j k} p_{k}^{\nu}
\end{aligned}
$$

where $H=G^{-1}$ is the inverse of the 3 by 3 Gram matrix defined by the momenta $p_{1}, p_{2}, p_{3}$. In terms of Mandelstam variables, these matrices are given by

$$
G=\left(\begin{array}{ccc}
0 & s & u \\
s & 0 & t \\
u & t & 0
\end{array}\right), \quad H=\frac{1}{2}\left(\begin{array}{ccc}
1 / s+1 / u & 1 / s & 1 / u \\
1 / s & 1 / s+1 / t & 1 / t \\
1 / u & 1 / t & 1 / t+1 / u
\end{array}\right) .
$$

$\mathcal{P}$ is a projector onto the $(n-3)$ dimensional subspace perpendicular to the 3 dimensional space spanned by the vectors $p_{1}, p_{2}, p_{3}, p_{4}$. $\mathcal{R}_{j, \nu}$ is the dual vector to $p_{j}^{\nu}$ relative to this 3 dimensional space. One may easily check the following relations for the objects $\mathcal{P}_{\mu \nu}$ and $\mathcal{R}_{j}^{\mu}$,

$$
\begin{aligned}
\mathcal{P}^{\mu \rho} \mathcal{P}_{\rho}{ }^{\nu} & =\mathcal{P}^{\mu \nu}, \\
\mathcal{P}^{\mu}{ }_{\nu} p_{j}^{\nu} & =0, \\
\mathcal{P}^{\mu}{ }_{\mu} & =\operatorname{tr}(\mathcal{P})=\operatorname{tr}(\mathcal{P} \cdot \mathcal{P})=n-3, \\
\mathcal{P}^{\mu}{ }_{\nu} \mathcal{R}_{j}^{\nu} & =0 \\
\mathcal{R}_{j, \nu} p_{k}^{\nu} & =\delta_{j k}, \\
\mathcal{R}_{j, \nu} \mathcal{R}_{l}{ }^{\nu} & =2 H_{j l} .
\end{aligned}
$$

To determine the tensor coefficients, $A_{j}$, we define the following linear operators

$$
\begin{aligned}
& \tilde{A}_{1}(\mathcal{M})=\frac{1}{(n-1)(n-3)} \mathcal{P}_{\mu_{1} \mu_{2}} \mathcal{P}_{\mu_{3} \mu_{4}} \mathcal{M}^{\mu_{1} \mu_{2} \mu_{3} \mu_{4}}, \\
& \tilde{A}_{2}(\mathcal{M})=\frac{1}{(n-1)(n-3)} \mathcal{P}_{\mu_{1} \mu_{3}} \mathcal{P}_{\mu_{2} \mu_{4}} \mathcal{M}^{\mu_{1} \mu_{2} \mu_{3} \mu_{4}}, \\
& \tilde{A}_{3}(\mathcal{M})=\frac{1}{(n-1)(n-3)} \mathcal{P}_{\mu_{1} \mu_{4}} \mathcal{P}_{\mu_{2} \mu_{3}} \mathcal{M}^{\mu_{1} \mu_{2} \mu_{3} \mu_{4}}
\end{aligned}
$$


One finds by direct calculation that

$$
\left(\begin{array}{c}
\tilde{A}_{1}(\mathcal{M}) \\
\tilde{A}_{2}(\mathcal{M}) \\
\tilde{A}_{3}(\mathcal{M})
\end{array}\right)=\frac{\operatorname{tr}(\mathcal{P})}{(n-1)(n-3)}\left(\begin{array}{ccc}
\operatorname{tr}(\mathcal{P}) & 1 & 1 \\
1 & \operatorname{tr}(\mathcal{P}) & 1 \\
1 & 1 & \operatorname{tr}(\mathcal{P})
\end{array}\right)\left(\begin{array}{l}
A_{1}(s, t, u) \\
A_{2}(s, t, u) \\
A_{3}(s, t, u)
\end{array}\right) .
$$

Staying in $n$-dimensions and inverting the system of equations yields,

$$
\left(\begin{array}{l}
A_{1}(s, t, u) \\
A_{2}(s, t, u) \\
A_{3}(s, t, u)
\end{array}\right)=\frac{1}{(n-4)}\left(\begin{array}{ccc}
(n-2) & -1 & -1 \\
-1 & (n-2) & -1 \\
-1 & -1 & (n-2)
\end{array}\right)\left(\begin{array}{c}
\tilde{A}_{1}(\mathcal{M}) \\
\tilde{A}_{2}(\mathcal{M}) \\
\tilde{A}_{3}(\mathcal{M})
\end{array}\right)
$$

We see that the right hand side appears to introduce an additional factor of $1 /(n-4)$. On the other hand, the sum

$$
A_{1}(s, t, u)+A_{2}(s, t, u)+A_{3}(s, t, u)=\tilde{A}_{1}(\mathcal{M})+\tilde{A}_{1}(\mathcal{M})+\tilde{A}_{1}(\mathcal{M})
$$

is free of spurious poles. We will discuss how these apparent extra poles cancel each other directly in the helicity amplitudes below.

It is straightforward to define projectors for the coefficients $B_{l k}^{\alpha}$,

$$
\begin{aligned}
\tilde{B}_{k l}^{1}(\mathcal{M})= & \frac{1}{(n-3)} \mathcal{P}_{\mu_{1} \mu_{2}} \mathcal{R}_{k \mu_{3}} \mathcal{R}_{l \mu_{4}} \mathcal{M}^{\mu_{1} \mu_{2} \mu_{3} \mu_{4}} \\
& -\frac{2}{(n-4)} H_{k l}\left((n-2) \tilde{A}_{1}(\mathcal{M})-\tilde{A}_{2}(\mathcal{M})-\tilde{A}_{3}(\mathcal{M})\right)
\end{aligned}
$$

where the last term is proportional to $A_{1}$. The other $B^{\alpha}$ 's are found by permuting the Lorentz indices in (3.23) and subtracting the respective $A$ 's. Acting with Eq. (3.23) on the general tensor one finds the desired property,

$$
\tilde{B}_{k l}^{\alpha}(\mathcal{M})=B_{k l}^{\alpha}(s, t, u)
$$

by using relations (3.14-3.19).

In the same spirit one can construct projectors for the coefficients $C_{j k l m}$,

$$
\begin{aligned}
\tilde{C}_{j k l m}(\mathcal{M})= & \mathcal{R}_{j \mu_{1}} \mathcal{R}_{k \mu_{2}} \mathcal{R}_{l \mu_{3}} \mathcal{R}_{m \mu_{4}} \mathcal{M}^{\mu_{1} \mu_{2} \mu_{3} \mu_{4}} \\
- & 2\left[H_{j k} \tilde{B}_{l m}^{1}(\mathcal{M})+H_{j l} \tilde{B}_{k m}^{2}(\mathcal{M})+H_{j m} \tilde{B}_{k l}^{3}(\mathcal{M})\right. \\
& \left.+H_{k l} \tilde{B}_{j m}^{4}(\mathcal{M})+H_{k m} \tilde{B}_{j l}^{5}(\mathcal{M})+H_{l m} \tilde{B}_{j k}^{6}(\mathcal{M})\right] \\
- & 4\left[\frac{H_{j k} H_{l m}}{(n-4)}\left((n-2) \tilde{A}_{1}(\mathcal{M})-\tilde{A}_{2}(\mathcal{M})-\tilde{A}_{3}(\mathcal{M})\right)\right. \\
& +\frac{H_{j l} H_{k m}}{(n-4)}\left((n-2) \tilde{A}_{2}(\mathcal{M})-\tilde{A}_{3}(\mathcal{M})-\tilde{A}_{1}(\mathcal{M})\right) \\
& \left.+\frac{H_{j m} H_{k l}}{(n-4)}\left((n-2) \tilde{A}_{3}(\mathcal{M})-\tilde{A}_{1}(\mathcal{M})-\tilde{A}_{2}(\mathcal{M})\right)\right],
\end{aligned}
$$


where the last three terms are proportional to $A_{1}, A_{2}$ and $A_{3}$ respectively and which again satisfies,

$$
\tilde{C}_{i j k l}(\mathcal{M})=C_{i j k l}(s, t, u) .
$$

The linear operators acting on the amplitude defined in Eqs. (3.20), (3.23) and (3.24) are sufficient to determine any of the coefficients on the right hand side of the general tensor decomposition of the amplitude in Eq. (3.3). The linear nature ensures that one can apply these projectors on a graph by graph basis.

All of the tensor coefficients are linearly related to the basis set of Eq. (3.6) by gauge invariance and permutation symmetry. An important and non-trivial check of a full diagrammatic calculation is to evaluate each of the tensor coefficients and to verify the relations amongst them imposed by the Ward identities.

It remains to be shown that the poles present in Eq. (3.22) cancel. This is seen by focussing on the dangerous $\tilde{A}_{j}$ terms only. For $C_{2111}$ one finds that the $1 /(n-4)$ pole drops out directly,

$$
\begin{aligned}
C_{2111}(s, t, u) & =\tilde{C}_{2111}(\mathcal{M}) \\
& =\ldots+4 H_{11} H_{12}\left[A_{1}(s, t, u)+A_{2}(s, t, u)+A_{3}(s, t, u)\right] .
\end{aligned}
$$

For $B_{11}^{1}$ one has,

$$
B_{11}^{1}(s, t, u)=\tilde{B}_{11}^{1}(\mathcal{M})=\ldots+\frac{t}{s u} A_{1}(s, t, u) .
$$

Using the Bose symmetry relations,

$$
A_{1}(s, t, u)=A_{1}(s, u, t), A_{2}(s, t, u)=A_{1}(u, t, s), A_{3}(s, t, u)=A_{1}(t, s, u),
$$

a short calculation shows that in Eqs. (3.8), (3.9) and (3.10) only the sum of the $A_{j}$ 's is present so that the helicity amplitudes are free of spurious poles. This ensures that the projector method can be applied on a graph by graph basis without the need to expand one order higher in $\epsilon$.

\section{Light-by-light scattering in SUSY QED}

\subsection{The SUSY Ward Identity}

Supersymmetric amplitudes are expected to satisfy the Supersymmetric Ward identity $[29,30,31]$,

$$
0=\left\langle 0\left|\left[Q, a_{1}^{\dagger} \ldots a_{n}^{\dagger}\right]\right| 0\right\rangle,
$$

where $Q$ is the Supersymmetry generator that satisfies $Q|0\rangle=0$ and $a_{i}^{\dagger}$ are the creation operators for particles in the initial state. Application of Eq. (4.1) to states with three photons and a photino yields the following identities,

$$
\begin{aligned}
& \mathcal{M}_{++++} \equiv 0, \\
& \mathcal{M}_{+++-} \equiv 0
\end{aligned}
$$


At tree level these identities are trivially satisfied due to the abelian nature of the photon. Beyond tree-level, couplings with the matter multiplet give rise to nontrivial interactions, that we investigate in the subsequent subsections. As mentioned in the introduction, we work in conventional dimensional regularisation and treat all momenta and Lorentz indices as $n$-dimensional. This breaks supersymmetry because the fermionic and bosonic degrees of freedom for the gauge multiplet are no longer equivalent. We therefore expect that there will be non-trivial corrections to the SUSY Ward identity that vanish in the 4-dimensional limit.

For convenience we decompose the helicity amplitudes perturbatively as

$$
\mathcal{M}_{\lambda_{1} \lambda_{2} \lambda_{3} \lambda_{4}}=\alpha^{2}\left(\mathcal{M}_{\lambda_{1} \lambda_{2} \lambda_{3} \lambda_{4}}^{(1)}+\frac{\alpha}{\pi} \mathcal{M}_{\lambda_{1} \lambda_{2} \lambda_{3} \lambda_{4}}^{(2)}+\mathcal{O}\left(\alpha^{2}\right)\right)
$$

\subsection{One-loop SUSY QED helicity amplitudes}

Because of the abelian nature of QED, there is no quartic photon coupling at tree level. At one-loop a four-point contribution is generated by the interaction of the photon with the matter multiplet. The one-loop amplitude can be decomposed according to gauge invariant subsets of graphs. There are two such groups at oneloop so that

$$
\mathcal{M}^{(1)}=\mathcal{M}^{(1), S}+\mathcal{M}^{(1), F},
$$

where the dependence on the helicities has been suppressed. These two contributions, $\mathcal{M}^{(1), S}$ and $\mathcal{M}^{(1), F}$, denote the scalar electron and electron loops respectively. In the Standard Model, only the electron loops, $\mathcal{M}^{(1), F}$, contribute. We find

$$
\begin{aligned}
\mathcal{M}_{++++}^{(1), S}= & 8+\mathcal{O}(\epsilon), \\
\mathcal{M}_{++++}^{(1), F}= & -8+\mathcal{O}(\epsilon), \\
\mathcal{M}_{+++-}^{(1), S}= & -8+\mathcal{O}(\epsilon), \\
\mathcal{M}_{+++-}^{(1), F}= & 8+\mathcal{O}(\epsilon), \\
\mathcal{M}_{++--}^{(1), S}= & 4-2\left((X-Y)^{2}+\pi^{2}\right)+4\left((X-Y)^{2}+\pi^{2}-2(X-Y)\right) \frac{t^{2}}{s^{2}} \\
& +\{u \leftrightarrow t\}+\mathcal{O}(\epsilon), \\
\mathcal{M}_{++--}^{(1), F}= & -4-4\left((X-Y)^{2}+\pi^{2}-2(X-Y)\right) \frac{t^{2}}{s^{2}} \\
& +\{u \leftrightarrow t\}+\mathcal{O}(\epsilon),
\end{aligned}
$$

where

$$
X=\log \left(\frac{-t}{s}\right), \quad Y=\log \left(\frac{-u}{s}\right) .
$$

Combining all graphs together, the one-loop light-by-light scattering amplitudes in SUSY QED are rather compact and are given by,

$$
\mathcal{M}_{++++}^{(1)} \equiv 0
$$




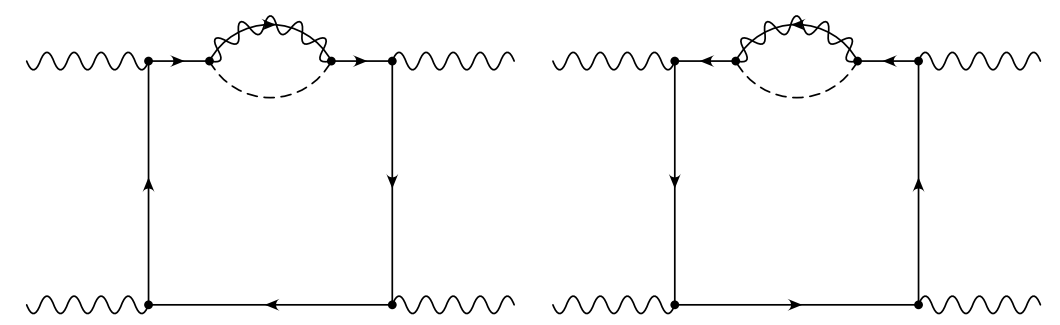

Figure 1: Typical diagrams involving the photino that are related by charge conjugation. The dashed line represents the scalar electron, while the overlaid solid and wavy lines represent the photino. Similar graphs can be drawn where the dashed line represents the scalar photon and the overlaid solid and wavy lines represent the electron.

$$
\begin{aligned}
\mathcal{M}_{+++-}^{(1)} & \equiv 0 \\
\mathcal{M}_{++--}^{(1)} & \equiv 4 s(n-4)\left(\operatorname{Box}^{6}(s, t)+\operatorname{Box}^{6}(s, u)\right)-4 s(n-2) \operatorname{Box}^{6}(t, u) \\
& =-4\left((X-Y)^{2}+\pi^{2}\right)+\mathcal{O}(\epsilon)
\end{aligned}
$$

where $\operatorname{Box}^{6}(s, t)$ is the (infrared and ultraviolet finite) one-loop box graph in $6-2 \epsilon$ dimensions. At one-loop there is no contribution from the gauge multiplet and this is therefore the result for both $N=1$ and $N=2$ SUSY QED.

The fact that $\mathcal{M}_{++++}^{(1)}$ and $\mathcal{M}_{+++-}^{(1)}$ both vanish is directly attributable to the SUSY Ward identity. The zeroes for $\mathcal{M}_{++++}^{(1)}$ and $\mathcal{M}_{+++-}^{(1)}$ occur at the level of the master integrals, i.e. to all orders in $n-4$. This is perhaps not surprising since although dimensional regularisation breaks the supersymmetry for the gauge multiplet, the photon and photino are not present as internal particles in any of the one-loop graphs. At this order, we are not sensitive to the SUSY breaking engendered by dimensional regularisation.

At one-loop we expect that amplitudes contain terms of weight 0,1 and 2 (counting logarithms and $\pi$ as weight 1 , squares of logarithms as weight 2 and constants (excluding $\pi$ ) as weight 0). By inspection of Eq. (4.6), we see that all such terms are present in the fermion and scalar contributions. However, the supersymmetric cancellation is such that only terms of weight 2 remain in Eq. (4.8), all weight 0 and weight 1 contributions are eliminated. We also note that terms proportional to ratios of the kinematic scales $t^{2} / s^{2}$ in the individual contributions (4.6) completely cancel in the supersymmetric result of Eq. (4.8).

\subsection{Two-loop SUSY QED helicity amplitudes}

Unlike the one-loop case, the chiral photino-electron-selectron coupling is present and it is necessary to address the issue of how to treat $\gamma_{5}$. Because of the parity invariance of the process, the scattering amplitude contains no antisymmetric part, indicating that the $\gamma_{5}$ contributions drop out. We can see this by considering a typical pair of diagrams with a photino exchange that are related by charge conjugation as shown 
in Fig. 1. Up to overall factors, the contribution from the first diagram is given by

$$
I_{1} \sim \int \frac{d^{n} k}{(2 \pi)^{n}} \frac{d^{n} \ell}{(2 \pi)^{n}} \frac{\operatorname{Tr}\left(P_{R} \ell P_{L} k \varepsilon_{1} k_{1} \varepsilon_{2} k_{12} \varepsilon_{3} k_{123} \varepsilon_{4} k\right)}{\left(k^{2}\right)^{2}\left(k_{1}\right)^{2}\left(k_{12}\right)^{2}\left(k_{123}\right)^{2} \ell^{2}(k-\ell)^{2}}
$$

where $k_{i \ldots j}=k+p_{i}+\ldots+p_{j}$ and

$$
P_{L}=\left(\frac{1-\gamma_{5}}{2}\right), \quad P_{R}=\left(\frac{1+\gamma_{5}}{2}\right)
$$

Similarly the second graph is

$$
I_{2} \sim \int \frac{d^{n} k}{(2 \pi)^{n}} \frac{d^{n} \ell}{(2 \pi)^{n}} \frac{\operatorname{Tr}\left(P_{R} \ell P_{L} k \varepsilon_{4} k_{4} \varepsilon_{3} k_{34} \varepsilon_{2} / k_{234} \varepsilon_{1} k\right)}{\left(k^{2}\right)^{2}\left(k_{4}\right)^{2}\left(k_{34}\right)^{2}\left(k_{234}\right)^{2} \ell^{2}(k-\ell)^{2}} .
$$

Relabelling $k \rightarrow-k, \ell \rightarrow-\ell$ and using charge conjugation to reverse the trace, we find that,

$$
I_{2} \sim \int \frac{d^{n} k}{(2 \pi)^{n}} \frac{d^{n} \ell}{(2 \pi)^{n}} \frac{\operatorname{Tr}\left(P_{L} \ell P_{R} k \varepsilon_{1} k_{1} \varepsilon_{2} k_{12} \varepsilon_{3} k_{123} \varepsilon_{4} k\right)}{\left(k^{2}\right)^{2}\left(k_{1}\right)^{2}\left(k_{12}\right)^{2}\left(k_{123}\right)^{2} \ell^{2}(k-\ell)^{2}}
$$

so that, up to common factors

$$
I_{1}+I_{2} \sim\left[P_{R} \ell P_{L}+P_{L} \ell P_{R}\right] \ldots=\frac{1}{2}\left[\ell-\gamma_{5} \ell \gamma_{5}\right] \ldots
$$

As expected, traces with single $\gamma_{5}$ factors drop out entirely. Since the amplitudes are finite, we therefore use an anti-commuting $\gamma_{5}$ such that,

$$
\frac{1}{2}\left[\ell-\gamma_{5} \ell \gamma_{5}\right]=\ell
$$

Similar arguments apply to the chiral couplings of the scalar photon with the electron.

\subsection{1 $N=1$ SUSY QED}

As in the one-loop case, it is convenient to break the amplitude up according to the different gauge-invariant pieces so that

$$
\mathcal{M}^{(2)}=\mathcal{M}^{(2), S}+\mathcal{M}^{(2), F}+\mathcal{M}^{(2), P}+\mathcal{M}^{(2), V}
$$

where the dependence on the helicities has been suppressed. At two-loops there are contributions from photino exchange, $\mathcal{M}^{(2), P}$, and the four scalar vertex, $\mathcal{M}^{(2), V}$, as well as graphs where the electron or scalar electron couple directly to the photons, $\mathcal{M}^{(2), F}$ and $\mathcal{M}^{(2), S}$. Altogether there are 1902 Feynman graphs which we generate using QGRAF[34]. Figures 2, 3, 4, 5 and 6 show the types of Feynman graphs relevant for the various contributions. Tadpole graphs and self-energies of external legs are not shown since they vanish in dimensional regularisation. We note that all of the possible diagrams are planar. 


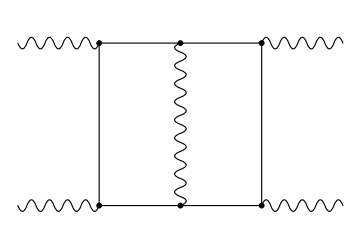

A

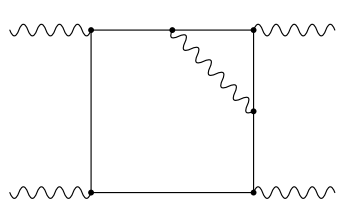

B

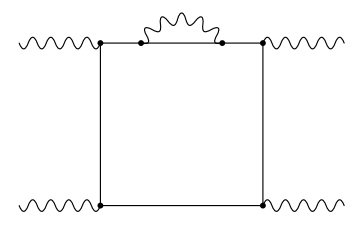

C

Figure 2: Graphs relevant for the electron, photino, scalar electron and scalar photon contributions $\mathcal{M}^{(2), F}, \mathcal{M}^{(2), P}, \mathcal{M}^{(2), S}$ and $\mathcal{M}^{(2), X}$. The solid lines represent particles from the matter multiplets, i.e. the electron or scalar electron while the internal wavy lines denote particles from the gauge multiplets, the photon, the photino or the scalar photon.

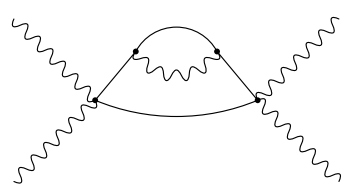

$\mathrm{D}$

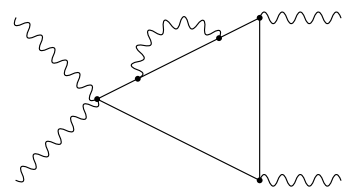

G

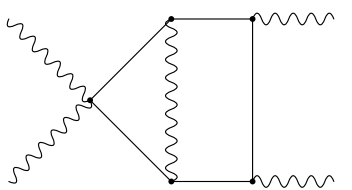

$\mathrm{E}$

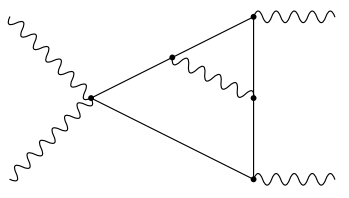

$\mathrm{H}$

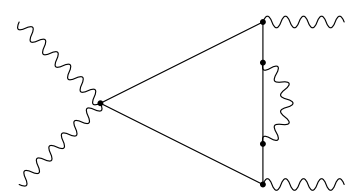

$\mathrm{F}$

Figure 3: Graphs relevant for the photino and scalar electron contributions $\mathcal{M}^{(2), P}$ and $\mathcal{M}^{(2), S}$. The solid lines represent the scalar electron while the wavy lines denote either the photon or photino.

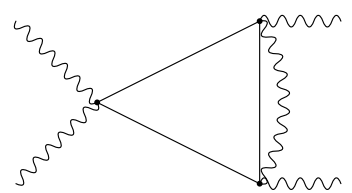

I

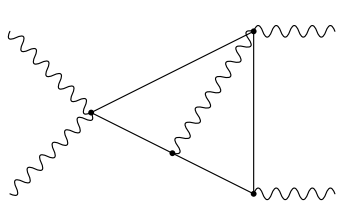

$\mathrm{L}$

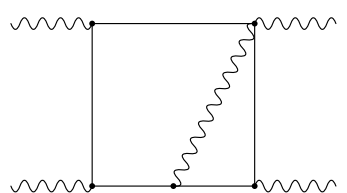

$\mathrm{O}$

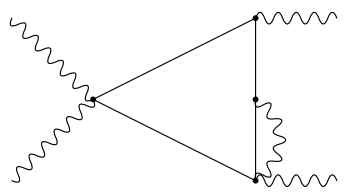

J

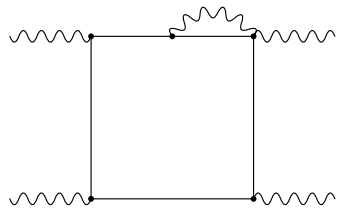

M

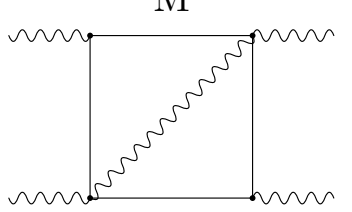

P

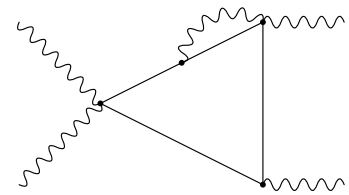

K
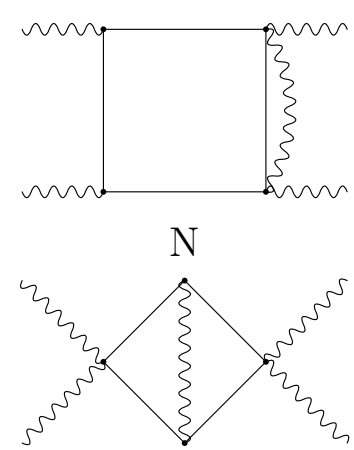

Q

Figure 4: Graphs relevant for the scalar electron contribution $\mathcal{M}^{(2), S}$. The solid lines represent the scalar electron while the wavy lines denote the photon. 


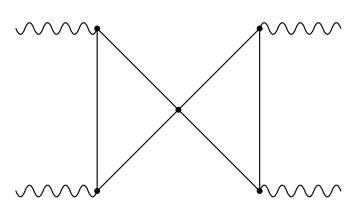

$\mathrm{R}$

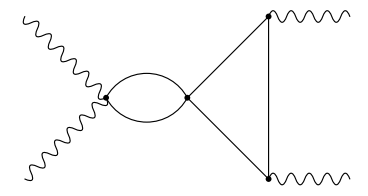

S

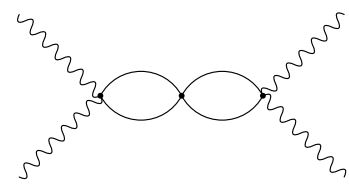

$\mathrm{T}$

Figure 5: Graphs relevant for the scalar four-point interaction contribution $\mathcal{M}^{(2), V}$. The solid lines represent the scalar electron while the wavy lines denotes the photon.

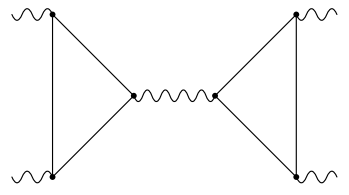

$\mathrm{U}$

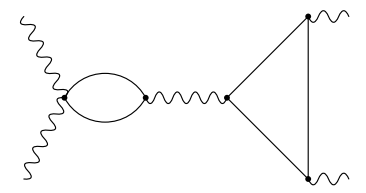

V

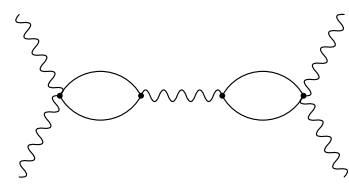

W

Figure 6: One-particle reducible graphs relevant for the electron, scalar electron and scalar photon contributions $\mathcal{M}^{(2), F}, \mathcal{M}^{(2), S}$ and $\mathcal{M}^{(2), X}$. The solid lines represent particles from the matter multiplet, i.e. the electron or scalar electron while the wavy lines denote the photon or scalar photon. Graphs $V$ and $W$ are only part of the scalar electron contribution.

Explicit evaluation of the two-loop graphs yields rather lengthy results for the individual gauge invariant subsets, and we list them in Appendix B. Combining the various components according to Eq. (4.15) gives the following two-loop $N=1$ SUSY QED helicity amplitudes,

$$
\begin{aligned}
\mathcal{M}_{++++}^{(2)}= & +\mathcal{O}(\epsilon), \\
\mathcal{M}_{+++-}^{(2)}= & +\mathcal{O}(\epsilon), \\
\mathcal{M}_{++--}^{(2)}= & +\left(-16 \mathrm{Li}_{4}(y)+8(Y+2) \mathrm{Li}_{3}(y)+8 Y \mathrm{Li}_{3}(x)\right. \\
& +8(X Y-Y+X) \mathrm{Li}_{2}(y)-16 \zeta_{3}+\frac{16}{45} \pi^{4}-\frac{2}{3}(6 Y+11+2 X Y) \pi^{2} \\
& \left.+\frac{2}{3}\left(-X^{4}+6 X^{2} Y^{2}+4 X Y^{3}+12 X Y-4 X^{3}+8 Y^{3}\right)\right) \\
& +\left(-16 \mathrm{Li}_{4}(z)-8(Y-X) \mathrm{Li}_{3}(y)-8(Y-X) \mathrm{Li}_{3}(x)-8 X Y \mathrm{Li}_{2}(y)\right. \\
& +\left(-\frac{8}{3} \pi^{2}-8 X Y\right) \mathrm{Li}_{2}(x)+\frac{1}{15} \pi^{4}-\frac{2}{3}(Y+2-X)(Y-X) \pi^{2} \\
& +\frac{1}{3}\left(4 X^{3}+12 X^{3} Y+12 X Y^{2}-12 X^{2} Y-4 X Y^{3}+Y^{4}-3 X^{4}\right. \\
& \left.\left.-30 X^{2} Y^{2}-4 Y^{3}\right)\right) \frac{t^{2}}{s^{2}}+8 X^{2} \frac{t}{u} \\
& +i \pi\left\{16 X \frac{t}{u}+\left(\frac{4}{3}(Y-X) \pi^{2}+\frac{4}{3}(Y-X)^{3}\right) \frac{t^{2}}{s^{2}}\right. \\
& +(Y)
\end{aligned}
$$




$$
\begin{aligned}
& +\left(16 \operatorname{Li}_{3}(x)+8 X \mathrm{Li}_{2}(y)+8 X \mathrm{Li}_{2}(x)\right. \\
& \left.\left.-\frac{4}{3}(2 Y-1) \pi^{2}-\frac{4}{3} Y\left(6 X-6 Y+Y^{2}-9 X Y-12\right)\right)\right\} \\
& +\{u \leftrightarrow t\}+\mathcal{O}(\epsilon)
\end{aligned}
$$

where we have used the standard polylogarithm identities [35] to express our results in terms of a basis set of constants (where $\zeta_{n}$ is the Riemann Zeta function, $\zeta_{2}=\pi^{2} / 6$, $\left.\zeta_{3}=1.202056 \ldots\right)$, logarithms and polylogarithms $\operatorname{Li}_{n}(w)$ defined by

$$
\begin{aligned}
& \operatorname{Li}_{n}(w)=\int_{0}^{w} \frac{d t}{t} \operatorname{Li}_{n-1}(t) \quad \text { for } n=2,3,4 \\
& \operatorname{Li}_{2}(w)=-\int_{0}^{w} \frac{d t}{t} \log (1-t) .
\end{aligned}
$$

with arguments $x, 1-x$ and $(x-1) / x$, where

$$
x=-\frac{t}{s}, \quad y=-\frac{u}{s}=1-x, \quad z=-\frac{u}{t}=\frac{x-1}{x} .
$$

In the physical region $s>0$ and $t, u<0$, our basis set of functions are all real.

As expected from the SUSY Ward identities, Eqs. (4.2) and (4.3), two of the helicity amplitudes vanish in the $n \rightarrow 4$ limit. However, the identities are violated by terms of $\mathcal{O}(\epsilon)$ which can be traced back to the SUSY breaking nature of dimensional regularisation.

The SUSY Ward identity does not require that $\mathcal{M}_{++--}$vanishes, but, as in the one-loop case, there are still significant cancellations and the full amplitude is somewhat more compact than the individual contributions (which are listed in Appendix B): In fact, at two-loops we expect that amplitudes contain terms up to weight 4 (counting $\operatorname{Li}_{n}, \ln ^{n}$ and $\zeta_{n}$ as weight $n, \ln ^{m} \mathrm{Li}_{n}$ as weight $n+m$ and so on). Each of the individual contributions for $\mathcal{M}_{++--}$listed in Appendix B, and in particular the fermion contribution $\mathcal{M}_{++--}^{(2), F}$ that corresponds to the Standard Model ( $N=0$ SUSY), demonstrates that all possible weights, $0, \ldots, 4$, are present. However, we see that the $N=1$ SUSY amplitude of Eq. (4.16) contains terms of weight 2, 3 and 4 and that, as in the one-loop case, terms of weight 0 and 1 cancel. We also note that the individual contributions contain terms proportional to the dimensionless ratios, $t^{4} / u^{2} s^{2}, t^{2} / s^{2}, t / u$ (and $t \leftrightarrow u$ ), while in the combination selected by $N=1$ SUSY, (4.16), the terms proportional to $t^{4} / u^{2} s^{2}$ drop out.

\subsection{2 $N=2$ SUSY QED}

For $N=2$ SUSY, there are additional contributions from the gaugino, the scalar photon and the modified quartic scalar electron interactions. In terms of the different 
gauge-invariant pieces we find that

$$
\mathcal{M}^{(2)}=\mathcal{M}^{(2), S}+\mathcal{M}^{(2), F}+2 \mathcal{M}^{(2), P}+3 \mathcal{M}^{(2), V}+\mathcal{M}^{(2), X},
$$

where again the dependence on the helicities has been suppressed. Here, $\mathcal{M}^{(2), X}$ denotes the 144 graphs involving the scalar photon while the factors of 2 and 3 multiplying the photino and four-point scalar electron contributions respectively reflect the aditional photino and the modified scalar electron interactions of the $N=2$ SUSY theory.

Combining the individual gauge invariant contributions listed in Appendix B according to Eq. (4.19), we find the $N=2$ SUSY QED helicity amplitudes are rather compact and are given by,

$$
\begin{aligned}
\mathcal{M}_{++++}^{(2)}= & +\mathcal{O}(\epsilon), \\
\mathcal{M}_{+++-}^{(2)}= & +\mathcal{O}(\epsilon), \\
\mathcal{M}_{++--}^{(2)}= & \left(-16 \operatorname{Li}_{4}(y)+8 Y \operatorname{Li}_{3}(x)+8 Y \operatorname{Li}_{3}(y)+\frac{16}{45} \pi^{4}-\frac{2}{3} X Y \pi^{2}\right. \\
& \left.-\frac{2}{3} Y^{3}(Y-4 X)\right) \\
& +i \pi\left\{\left(16 \operatorname{Li}_{3}(x)-\frac{4}{3} Y \pi^{2}-\frac{4}{3} Y^{2}(Y-3 X)\right)\right\} \\
& +\{u \leftrightarrow t\}+\mathcal{O}(\epsilon) .
\end{aligned}
$$

As expected, two of the helicity amplitudes vanish as $n \rightarrow 4$ due to the SUSY Ward identity. The remaning non-trivial helicity amplitude is considerably simpler than that obtained in either pure QED [1] (see the fermion loop contributions in Appendix B) or the $N=1$ SUSY QED helicity amplitudes of Eq. (4.16). In particular, we note that only terms of weight 4 remain, the contributions of weight 2 and 3 (that were present in the $N=1$ case) have cancelled. Furthermore, all terms depending on the ratios of kinematic scales have dropped out. 


\section{Summary}

We have demonstrated that the method based on $n$-dimensional projections is able to generate helicity amplitudes in an efficient way. We have been able to confirm the previous results for photon-photon scattering via a charged fermion loop obtained by helicity methods in the high energy limit where the fermion mass can be ignored. The method can, in principle, be used for more complicated processes, involving massive particles in the loop, non-abelian fields and/or more external vertices. The method is constructed such that one can use standard $n$-dimensional Lorentz covariant reduction techniques for tensor-integrals not only for squared matrix elements, but also for helicity amplitudes.

As an application we studied photon-photon scattering in the theoretically interesting cases of $N=1$ and $N=2$ supersymmetric QED. Because the process is both ultraviolet and infrared convergent, the results are explicitly supersymmetric in $n=4$, which is sufficient to draw conclusions regarding SUSY cancellations. We have not addressed the question of whether the method can be used to define supersymmetric amplitudes also at higher order in $(n-4)$. However, we expect that the situation here will be similar to that found using other techniques, where explicit terms proportional to $(n-4)$ have to be added in order to preserve the SUSY Ward identities at all orders in $(n-4)$.

The results we found have an interesting pattern of simplification as one increases the number of supersymmetries. In normal QED, corresponding to $N=0$ supersymmetries, one finds three independent amplitudes. For supersymmetric theories, two of these helicity amplitudes vanish. This is well understood on the basis of the supersymmetric Ward identity. An intricate pattern of cancellations occurs in the remaining (non-trivial) amplitude, $\mathcal{M}_{++--}$, as $N$ is increased, both in terms of the dimensionless ratios of kinematic scales and in the weights of the functions present in the amplitude.

First, considering the one-loop level, one finds in normal $(N=0)$ QED terms proportional to the dimensionless ratios $t^{2} / s^{2}$ and $u^{2} / s^{2}$ together with weights of logarithm of 0,1, 2 (see Eq. (4.6). In the supersymmetric case (4.8), there are no dimensionless ratios and there is a uniform weight of 2 for the logarithms.

\begin{tabular}{|c|r|r|}
\hline $\mathcal{M}_{++--}$ & one-loop & two-loop \\
\hline$N=0$ & $0,1,2$ & $0,1,2,3,4$ \\
$N=1$ & 2 & $2,3,4$ \\
$N=2$ & 2 & 4 \\
\hline
\end{tabular}

Table 1: Weights of terms contribution to $\mathcal{M}_{++--}$at one- and two-loops. The $N=0$ result refers to the fermion contribution of Eq. (B.3) while values given for $N=1$ and $N=2$ are extracted from Eqs. (4.16) and (4.20). 


\begin{tabular}{|l|c|c|c|}
\hline $\mathcal{M}_{++--}$ & 1 & $\frac{t^{2}}{s^{2}}, \frac{u^{2}}{s^{2}}, \frac{t}{u}, \frac{u}{t}$ & $\frac{t^{4}}{u^{2} s^{2}}, \frac{u^{4}}{t^{2} s^{2}}$ \\
\hline$N=0$ & $\sqrt{ }$ & $\sqrt{ }$ & $\sqrt{ }$ \\
$N=1$ & $\sqrt{ }$ & $\sqrt{ }$ & \\
$N=2$ & $\sqrt{ }$ & & \\
\hline
\end{tabular}

Table 2: Ratios of kinematic scales appearing in the two-loop amplitude $\mathcal{M}_{++--}$for different amounts of supersymmetry.

At the two-loop level, the pattern is similar, see Tables 1 and 2. Normal $(N=0)$ QED contains powers of $t / s$ up to 4 and all weights of logarithm from 0 to 4 . The $N=1$ case shows some simplifications, powers of $t / s$ exist only up to squares and the logarithmic terms have weights $2,3,4$. Finally in the $N=2$ theory there are no powers of $t / s$ in the amplitudes and the logarithms are uniformly of weight 4 . We see therefore, that increasing the number of supersymmetries reduces the complexity of the amplitudes and that the $N=2$ theory is maximally simplified. A conjecture would be that this pattern persists in higher orders of perturbation theory, where one would expect no powers and a weight of two times the level of loops in the logarithms. This pattern might be explainable by performing similar calculations in superspace.

\section{Acknowledgements}

We thank Valya Khoze, Adrian Signer, Bas Tausk and Georg Weiglein for helpful discussions. This work was supported in part by the UK Particle Physics and Astronomy Research Council, by the EU Fifth Framework Programme 'Improving $\mathrm{Hu}$ man Potential', Research Training Network 'Particle Physics Phenomenology at High Energy Colliders', contract HPRN-CT-2000-00149 and by the DFG-Forschergruppe Quantenfeldtheorie, Computeralgebra und Monte-Carlo Simulation. We thank the British Council and German Academic Exchange Service for support under ARC project 1050. PM acknowledges the support of the German Academic Exchange Service. 


\section{References}

[1] Z. Bern, A. De Freitas, L. J. Dixon, A. Ghinculov and H. L. Wong, JHEP 0111 (2001) 031 [arXiv:hep-ph/0109079].

[2] R. Karplus and M. Neuman, Phys. Rev. 80 (1950) 380

[3] R. Karplus and M. Neuman, Phys. Rev. 83 (1951) 776.

[4] B. De Tollis, Il Nuovo Cim. 32 (1964) 757.

[5] B. De Tollis, Il Nuovo Cim. 35 (1965) 1182.

[6] V. Costantini, B. De Tollis and G. Pistoni, Il Nuovo Cim. 2A, 3 (1971) 733.

[7] V. A. Smirnov, Phys. Lett. B 460 (1999) 397 [arXiv:hep-ph/9905323].

[8] V. A. Smirnov and O. L. Veretin, Nucl. Phys. B 566 (2000) 469 [arXiv:hep$\mathrm{ph} / 9907385]$.

[9] J. B. Tausk, Phys. Lett. B 469 (1999) 225 [arXiv:hep-ph/9909506].

[10] C. Anastasiou, T. Gehrmann, C. Oleari, E. Remiddi and J. B. Tausk, legs," Nucl. Phys. B 580 (2000) 577 [arXiv:hep-ph/0003261].

[11] C. Anastasiou, E. W. N. Glover and C. Oleari, Nucl. Phys. B 575 (2000) 416 [Erratumibid. B 585 (2000) 763] [arXiv:hep-ph/9912251].

[12] T. Gehrmann and E. Remiddi, Nucl. Phys. Proc. Suppl. 89 (2000) 251 [arXiv:hep$\mathrm{ph} / 0005232]$.

[13] C. Anastasiou, J. B. Tausk and M. E. Tejeda-Yeomans, Nucl. Phys. Proc. Suppl. 89 (2000) 262 [arXiv:hep-ph/0005328].

[14] K. G. Chetyrkin, A. L. Kataev and F. V. Tkachov, Nucl. Phys. B 174 (1980) 345.

[15] K. G. Chetyrkin and F. V. Tkachov, Nucl. Phys. B 192 (1981) 159.

[16] T. Gehrmann and E. Remiddi, Nucl. Phys. B 580 (2000) 485 [arXiv:hep-ph/9912329].

[17] Z. Bern, L. J. Dixon and D. A. Kosower, JHEP 0001 (2000) 027 [arXiv:hep$\mathrm{ph} / 0001001]$.

[18] Z. Bern, L. J. Dixon and A. Ghinculov, Phys. Rev. D 63 (2001) 053007 [arXiv:hep$\mathrm{ph} / 0010075]$.

[19] C. Anastasiou, E. W. N. Glover, C. Oleari and M. E. Tejeda-Yeomans, Nucl. Phys. B 601 (2001) 318 [arXiv:hep-ph/0010212].

[20] C. Anastasiou, E. W. N. Glover, C. Oleari and M. E. Tejeda-Yeomans, Nucl. Phys. B 601 (2001) 341 [arXiv:hep-ph/0011094]. 
[21] C. Anastasiou, E. W. N. Glover, C. Oleari and M. E. Tejeda-Yeomans, Nucl. Phys. B 605 (2001) 486 [arXiv:hep-ph/0101304].

[22] E. W. N. Glover, C. Oleari and M. E. Tejeda-Yeomans, Nucl. Phys. B 605 (2001) 467 [arXiv:hep-ph/0102201].

[23] Z. Bern, A. De Freitas and L. J. Dixon, JHEP 0109 (2001) 037 [arXiv:hep$\mathrm{ph} / 0109078]$.

[24] Z. Bern, A. De Freitas and L. Dixon, arXiv:hep-ph/0201161.

[25] C. Anastasiou, E. W. N. Glover and M. E. Tejeda-Yeomans, arXiv:hep-ph/0201274.

[26] J. Wess and B. Zumino, Nucl. Phys. B 78 (1974) 1.

[27] W. Hollik, E. Kraus and D. Stockinger, Eur. Phys. J. C 11 (1999) 365 [arXiv:hep$\mathrm{ph} / 9907393]$.

[28] P. Fayet, Nucl. Phys. B 113 (1976) 135.

[29] M. T. Grisaru, H. N. Pendleton and P. van Nieuwenhuizen, Phys. Rev. D 15 (1977) 996.

[30] M. T. Grisaru and H. N. Pendleton, Nucl. Phys. B 124 (1977) 81.

[31] S. J. Parke and T. R. Taylor, Phys. Lett. B 157 (1985) 81 [Erratum-ibid. 174B (1985) 465].

[32] Z. Bern, J. S. Rozowsky and B. Yan, Phys. Lett. B 401 (1997) 273 [arXiv:hepph/9702424].

[33] Z. Bern, L. J. Dixon, D. C. Dunbar, M. Perelstein and J. S. Rozowsky, divergences," Nucl. Phys. B 530 (1998) 401 [arXiv:hep-th/9802162].

[34] P. Nogueira, J. Comput. Phys. 105, 279 (1993).

[35] K.S. Kölbig, J.A. Mignaco and E. Remiddi, B.I.T. 10 (1970) 38. 


\section{A. The SUSY QED Feynman Rules}

The Feynman rules for the SUSY QED Lagrangians of Eqs. (2.1) and (2.3) with all momenta incoming are given by,
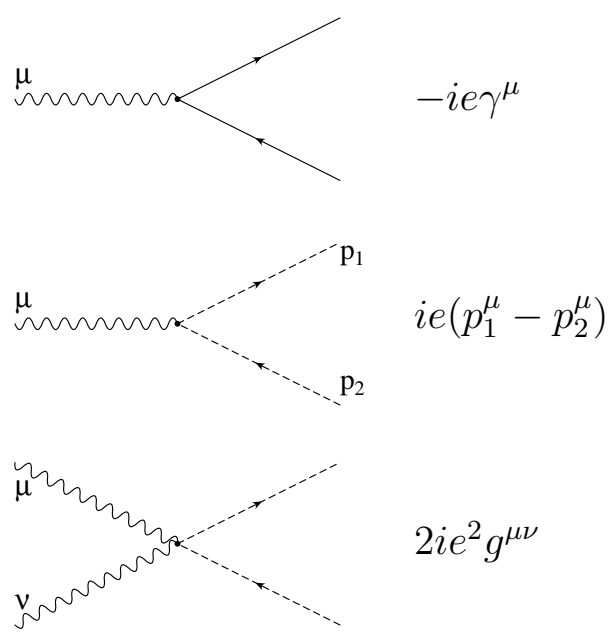

$2 i e^{2} g^{\mu \nu}$
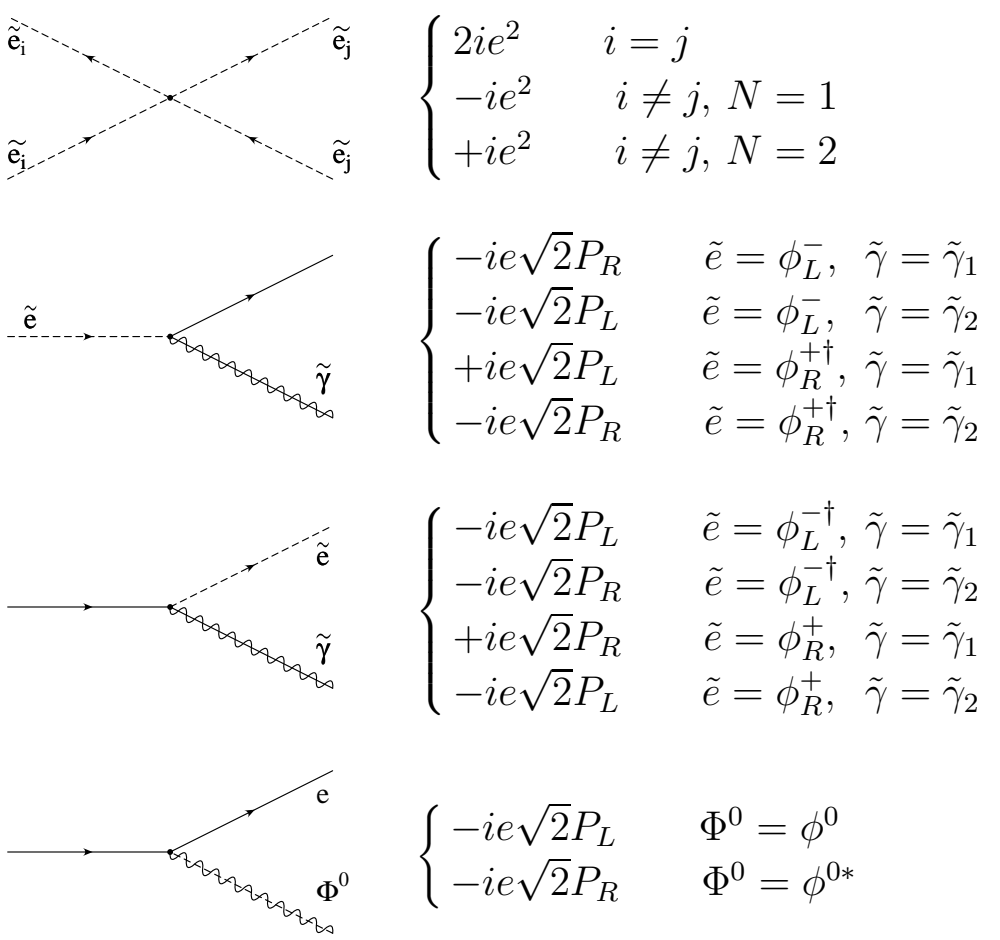

where the photon, electron, scalar electron, photino and scalar photon are denoted by wavy, solid, dashed, overlaid solid and wavy and overlaid dashed and wavy lines respectively. 


\section{B. Two-loop contributions to the helicity amplitudes}

In this appendix we list the individual contributions to the two-loop helicity amplitudes. There are five separate gauge invarariant contributions, loops with scalar particles coupling to photons, electron loops, photino exchange graphs, diagrams with the four scalar vertex and graphs involving the scalar photon. We find that individual two-loop contributions are given by,

$$
\begin{aligned}
& \mathcal{M}_{++++}^{(2), S}=-6 \\
& \mathcal{M}_{++++}^{(2), F}=-12 \\
& \mathcal{M}_{++++}^{(2), P}=+24 \\
& \mathcal{M}_{++++}^{(2), V}=-6 \\
& \mathcal{M}_{++++}^{(2), X}=-12 \\
& \mathcal{M}_{+++-}^{(2), S}=-4 X^{2} \frac{t^{4}}{u^{2} s^{2}}+\left(\pi^{2}-2\left(2 Y+X Y-3 X^{2}\right)\right)-4 X(2+3 X) \frac{t}{u} \\
& +\left(-2 \pi^{2}-2\left(2 Y-2 X-2 X Y-3 X^{2}+3 Y^{2}\right)\right) \frac{t^{2}}{s^{2}} \\
& +i \pi\left\{-8(1+3 X) \frac{t}{u}+4(-1+2 X)-8(-2 X+Y) \frac{t^{2}}{s^{2}}-8 X \frac{t^{4}}{u^{2} s^{2}}\right\} \\
& +\{u \leftrightarrow t\} \\
& \mathcal{M}_{+++-}^{(2), F}=-2 X^{2} \frac{t^{4}}{u^{2} s^{2}}-\left(-X^{2}+2 Y\right)-2 X(2+3 X) \frac{t}{u} \\
& +\left(-\pi^{2}-\left(2 Y-2 X-2 X Y-3 X^{2}+3 Y^{2}\right)\right) \frac{t^{2}}{s^{2}} \\
& +i \pi\left\{-4(1+3 X) \frac{t}{u}+2(X-1)-4(-2 X+Y) \frac{t^{2}}{s^{2}}-4 X \frac{t^{4}}{u^{2} s^{2}}\right\} \\
& +\{u \leftrightarrow t\} \\
& \mathcal{M}_{+++-}^{(2), P}=6 X^{2} \frac{t^{4}}{u^{2} s^{2}}+\left(-\pi^{2}+\left(6 Y-7 X^{2}+2 X Y\right)\right)+6 X(2+3 X) \frac{t}{u} \\
& +\left(3 \pi^{2}+3\left(2 Y-2 X-2 X Y-3 X^{2}+3 Y^{2}\right)\right) \frac{t^{2}}{s^{2}} \\
& +i \pi\left\{12(1+3 X) \frac{t}{u}-2(5 X-3)+12(-2 X+Y) \frac{t^{2}}{s^{2}}+12 X \frac{t^{4}}{u^{2} s^{2}}\right\} \\
& +\{u \leftrightarrow t\} \\
& \mathcal{M}_{+++-}^{(2), X}=-\mathcal{M}_{+++-}^{(2), P} \\
& \mathcal{M}_{+++-}^{(2), V}=0
\end{aligned}
$$




$$
\begin{aligned}
& \mathcal{M}_{++--}^{(2), S}=-4 X^{2} \frac{t^{4}}{u^{2} s^{2}}-8 X(X+1) \frac{t}{u} \\
& +\left(16 \operatorname{Li}_{4}(x)-8(Y-2) \operatorname{Li}_{3}(y)-8 \operatorname{Li}_{3}(x) Y-8(X-Y+X Y) \operatorname{Li}_{2}(x)\right. \\
& -16 \zeta_{3}-\frac{31}{45} \pi^{4}+\frac{4}{3}\left(-3 Y-3-X^{2}+2 X Y\right) \pi^{2} \\
& \left.+\frac{1}{3}\left(-24 X^{2}-3-18 X^{2} Y^{2}-48 X+8 Y^{3}+24 X Y\right)\right) \\
& +\left(-16 \operatorname{Li}_{4}(z)-32 \operatorname{Li}_{4}(y)-32 \operatorname{Li}_{4}(x)\right. \\
& +8(-2+Y+3 X) \operatorname{Li}_{3}(y)+8(Y+3 X+2) \operatorname{Li}_{3}(x)+8 \operatorname{Li}_{2}(y) X Y \\
& +\left(-\frac{8}{3} \pi^{2}+8(-2 Y-2 X+X Y)\right) \operatorname{Li}_{2}(x)+\frac{112}{45} \pi^{4} \\
& +\frac{4}{3}\left(-6 X Y+4 Y+Y^{2}+3-2 X+X^{2}\right) \pi^{2} \\
& -\frac{4}{3}\left(-9 X^{2}+X^{4}+6 X^{2} Y-9 X^{2} Y^{2}-4 X^{3} Y+6 X Y^{2}-6 Y-2 X^{3}+2 Y^{3}\right. \\
& +6 X+6 X Y)) \frac{t^{2}}{s^{2}} \\
& +i \pi\left\{-8(1+2 X) \frac{t}{u}-8 X \frac{t^{4}}{u^{2} s^{2}}\right. \\
& +\left(-16 \operatorname{Li}_{3}(y)-8 X \operatorname{Li}_{2}(y)-8 \operatorname{Li}_{2}(x) X+\frac{8}{3} \pi^{2} Y\right. \\
& \left.+\frac{4}{3}\left(6 Y^{2}-12-6 X Y-9 X Y^{2}+Y^{3}\right)\right) \\
& +\left(32 \mathrm{Li}_{3}(y)+32 \mathrm{Li}_{3}(x)+32 Y \mathrm{Li}_{2}(y)+32(Y-1) \mathrm{Li}_{2}(x)\right. \\
& -\frac{4}{3}(-2+5 Y+3 X) \pi^{2} \\
& \left.\left.-\frac{4}{3}\left(-9 X^{2} Y+6 Y^{2}-27 X Y^{2}+12 X Y+6 Y-12 X-6 X^{2}+Y^{3}+3 X^{3}\right)\right) \frac{t^{2}}{s^{2}}\right\} \\
& +\{u \leftrightarrow t\} \\
& \mathcal{M}_{++--}^{(2), F}=-2 X^{2} \frac{t^{4}}{u^{2} s^{2}} \\
& +\left(16 \operatorname{Li}_{3}(y)+8(Y-X) \operatorname{Li}_{2}(x)-16 \zeta_{3}-\frac{2}{3}(5+6 Y) \pi^{2}\right.
\end{aligned}
$$




$$
\begin{aligned}
& \left.+\frac{2}{3}\left(-6 Y^{2}+12 X Y+4 Y^{3}-3-12 Y\right)\right)-4 X \frac{t}{u} \\
& +\left(-16 \mathrm{Li}_{4}(z)-16 \mathrm{Li}_{4}(y)-16 \mathrm{Li}_{4}(x)+8(-1+2 X) \mathrm{Li}_{3}(y)\right. \\
& +8(1+2 X) \operatorname{Li}_{3}(x)+\left(-\frac{8}{3} \pi^{2}-8(X+Y)\right) \operatorname{Li}_{2}(x)+\frac{7}{9} \pi^{4} \\
& -\frac{2}{3}\left(Y^{2}+2 X Y-Y-X-3+X^{2}\right) \pi^{2}-\frac{1}{3}\left(5 X^{4}-6 X Y^{2}-12 Y-10 X^{3}+Y^{4}\right. \\
& \left.\left.-18 X^{2}-4 X Y^{3}-20 X^{3} Y+6 X^{2} Y^{2}+12 X Y+12 X+10 Y^{3}+30 X^{2} Y\right)\right) \frac{t^{2}}{s^{2}} \\
& +i \pi\left\{-4 \frac{t}{u}+\left(\frac{4}{3} \pi^{2}+8\left(-X Y+Y-1+Y^{2}\right)\right)-4 X \frac{t^{4}}{u^{2} s^{2}}\right. \\
& +\left(16 \operatorname{Li}_{3}(y)+16 \mathrm{Li}_{3}(x)+16 Y \mathrm{Li}_{2}(y)+16(Y-1) \mathrm{Li}_{2}(x)\right. \\
& -\frac{4}{3}(-1+2 Y+2 X) \pi^{2} \\
& \left.\left.+\frac{4}{3}\left(12 X Y^{2}-3 Y+6 X^{2} Y-3 Y^{2}-2 X^{3}+6 X+3 X^{2}-6 X Y\right)\right) \frac{t^{2}}{s^{2}}\right\} \\
& +\{u \leftrightarrow t\} \\
& \mathcal{M}_{++--}^{(2), P}=6 X^{2} \frac{t^{4}}{u^{2} s^{2}}+4 X(4 X+3) \frac{t}{u} \\
& +\left(-32 \operatorname{Li}_{4}(x)+16 \operatorname{Li}_{3}(y) Y+16(Y-1) \operatorname{Li}_{3}(x)+8(Y-X) \operatorname{Li}_{2}(y)\right. \\
& +16 \mathrm{Li}_{2}(x) Y X+16 \zeta_{3}+\frac{47}{45} \pi^{4}-\frac{2}{3}\left(6 X Y-2 X^{2}-6 X-5\right) \pi^{2} \\
& \left.-\frac{2}{3}\left(-15 X^{2} Y^{2}-18 Y^{2}+12 X Y-6-36 Y-4 X^{3} Y+Y^{4}+4 Y^{3}\right)\right) \\
& +\left(16 \mathrm{Li}_{4}(z)+48 \mathrm{Li}_{4}(y)+48 \mathrm{Li}_{4}(x)-8(-3+2 Y+4 X) \mathrm{Li}_{3}(y)\right. \\
& -8(2 Y+4 X+3) \operatorname{Li}_{3}(x)-16 \mathrm{Li}_{2}(y) X Y \\
& +\left(\frac{8}{3} \pi^{2}-8(-3 Y+2 X Y-3 X)\right) \operatorname{Li}_{2}(x)-\frac{16}{5} \pi^{4} \\
& -\frac{2}{3}\left(11 Y+2 Y^{2}-16 X Y-5 X+9+2 X^{2}\right) \pi^{2} \\
& +\frac{2}{3}\left(-18 Y+3 X^{4}+15 X Y^{2}-27 X^{2}-7 X^{3}+Y^{4}-30 X^{2} Y^{2}-4 X Y^{3}\right.
\end{aligned}
$$




$$
\begin{aligned}
& \left.\left.-12 X^{3} Y+18 X Y+18 X+7 Y^{3}+21 X^{2} Y\right)\right) \frac{t^{2}}{s^{2}} \\
& +i \pi\left\{4(3+8 X) \frac{t}{u}+12 X \frac{t^{4}}{u^{2} s^{2}}\right. \\
& +\left(32 \operatorname{Li}_{3}(x)+16 X \operatorname{Li}_{2}(y)+16 \operatorname{Li}_{2}(x) X-\frac{4}{3}(4 Y+1) \pi^{2}\right. \\
& \left.-\frac{8}{3}\left(-3 Y-9 X Y^{2}-3 X Y+Y^{3}+3 X^{2}-9\right)\right) \\
& +\left(-48 \mathrm{Li}_{3}(y)-48 \mathrm{Li}_{3}(x)-48 Y \mathrm{Li}_{2}(y)-48(Y-1) \mathrm{Li}_{2}(x)\right. \\
& +\frac{4}{3}(-3+8 Y+4 X) \pi^{2}+\frac{4}{3}\left(2 Y^{3}+9 Y+9 Y^{2}-18 X\right. \\
& \left.\left.\left.-12 X^{2} Y-42 X Y^{2}+4 X^{3}-9 X^{2}+18 X Y\right)\right) \frac{t^{2}}{s^{2}}\right\} \\
& +\{u \leftrightarrow t\} \\
& \mathcal{M}_{++--}^{(2), X}=-6 X^{2} \frac{t^{4}}{u^{2} s^{2}}-12 X(2 X+1) \frac{t}{u} \\
& +\left(32 \operatorname{Li}_{4}(y)-16 X \operatorname{Li}_{3}(x)-16 X \operatorname{Li}_{3}(y)-\frac{47}{45} \pi^{4}-\frac{2}{3}\left(-1+2 X^{2}\right) \pi^{2}\right. \\
& \left.+\frac{2}{3}\left(-3-4 X^{3} Y-18 Y^{2}-3 X^{2} Y^{2}+Y^{4}-36 Y\right)\right) \\
& +\left(-48 \operatorname{Li}_{4}(y)-48 \operatorname{Li}_{4}(x)+24(1+Y+X) \operatorname{Li}_{3}(x)+24(Y+X-1) \operatorname{Li}_{3}(y)\right. \\
& -24(Y+X) \operatorname{Li}_{2}(x)+\frac{47}{15} \pi^{4}+\frac{2}{3}\left(13 Y+3 X^{2}+9-7 X+3 Y^{2}-12 X Y\right) \pi^{2} \\
& -\frac{1}{3}\left(-12 X^{3} Y-36 Y+36 X-10 X^{3}+3 X^{4}-54 X^{2}+30 X^{2} Y+3 Y^{4}\right. \\
& \left.\left.+10 Y^{3}-12 X Y^{3}+42 X Y^{2}+36 X Y-18 X^{2} Y^{2}\right)\right) \frac{t^{2}}{s^{2}} \\
& +i \pi\left\{-12(1+4 X) \frac{t}{u}-12 X \frac{t^{4}}{u^{2} s^{2}}\right. \\
& +\left(-32 \operatorname{Li}_{3}(y)+\frac{8}{3} X \pi^{2}+\frac{8}{3}\left(-9+Y^{3}-3 X^{2} Y-9 Y\right)\right) \\
& +\left(48 \mathrm{Li}_{3}(x)+48 \mathrm{Li}_{3}(y)-48 \mathrm{Li}_{2}(x)-4(Y+X-1) \pi^{2}\right. \\
& \left.\left.-4\left(6 X Y+X^{3}-3 X^{2}-3 X Y^{2}-6 X-3 X^{2} Y+3 Y^{2}+3 Y+Y^{3}\right)\right) \frac{t^{2}}{s^{2}}\right\}
\end{aligned}
$$




$$
\begin{aligned}
+ & \{u \leftrightarrow t\} \\
\mathcal{M}_{++--}^{(2), V}= & -1
\end{aligned}
$$

The contribution from fermion exchange $\mathcal{M}^{(2), F}$ has previously been computed in Ref. [1] and we find complete agreement with the results presented there once the different definitions of the helicity amplitudes are taken into account. 